\title{
Abordagem da vida sexual feminina nos Cuidados de Saúde Primários
}

Lúcia Ramalheiro, ${ }^{*}$ Catarina Godinho, ${ }^{* *}$ Ana Catarina Maia***

\section{RESUMO}

Objectivo: Identificar obstáculos à abordagem da vida sexual nas consultas de Planeamento Familiar/Saúde da Mulher da Unidade de Saúde Familiar (USF) do Castelo, na perspectiva das utentes, a fim de desenvolver estratégias facilitadoras dessa mesma abordagem.

Tipo de Estudo: Estudo transversal, descritivo e observacional Local: USF do Castelo - Agrupamento de Centros de Saúde Seixal e Sesimbra

População: Mulheres com 18 ou mais anos frequentadoras das consultas de Planeamento familiar/Saúde da Mulher da USF do Castelo

Métodos: Aplicação de questionário auto-preenchido, anónimo e confidencial em consultas de planeamento familiar entre 15 de Março e 15 de Maio de 2010. Base de dados e tratamento estatístico realizados no Microsoft ${ }^{\circledR}$ Office Excel 2007.

Resultados: Foram analisados 161 questionários em que $5 \%$ das mulheres referiram ter problemas a nível sexual. Do total das analisadas, $76 \%$ consideram importante abordar a sexualidade na consulta médica, sendo que 19,3\% afirmam fazê-lo frequentemente. Relativamente às razões apontadas como barreiras à abordagem deste tema, destacam-se a vergonha por parte das utentes (42,5\%), o receio de que o médico não considere o tema importante (12,6\%) e a falta de tempo na consulta (10,3\%). Conclusões: Ao conhecermos as principais barreiras apontadas pelas utentes da USF do Castelo à inserção do tema vida sexual na consulta, concluímos que é necessário que o médico mostre que existe à-vontade, disponibilidade e necessidade de o fazer.

Palavras-chave: Sexualidade; Cuidados de Saúde Primários; Género Feminino.

\section{INTRODUÇÃO}

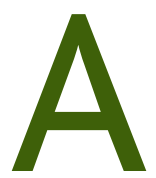
maioria das mulheres, segundo estudos efectuados nos Estados Unidos da América (EUA), afirma ser importante ter uma vida sexual satisfatória. ${ }^{1}$ No entanto, muitas têm problemas nesta área, os quais afectam a sua auto-estima, relacionamento com os parceiros e qualidade de vida. ${ }^{2,3}$ Segundo Laumann E.O. et al. $^{4}$, nos EUA, cerca de $43 \%$ mulheres entre os 18 e 59 anos apresentam disfunção sexual, sendo que apenas $20 \%$ destas procuram ajuda junto do seu médico de família. ${ }^{3,4,5}$ Este é portanto um problema pouco abordado, o que torna a disfunção sexual feminina uma patologia subdiagnosticada. $^{3,4,6}$

\footnotetext{
*Assistente de Medicina Geral e Familiar na USF Torre - ACES de Seixal-Sesimbra **Assistente de Medicina Geral e Familiar na UCSP Viso - ACES Setúbal-Palmela ***Médica Interna de Medicina Geral e Familiar na USF do Castelo - ACES de Seixal-Sesimbra
}

Quando questionadas acerca da abordagem de problemas relacionados com a sua vida sexual na consulta, muitas mulheres referem ter vergonha de falar sobre o tema e receio de que o médico se sinta desconfortável e menospreze o seu problema. ${ }^{1,2,7}$ Um estudo recente refere que apenas $14 \%$ das mulheres revela espontaneamente ter problemas na sua vida sexual e que $58 \%$ o faz somente quando esta questão está incluída em questionários. ${ }^{2,8}$ Por outro lado, os médicos também têm relutância em introduzir a problemática da sexualidade na consulta por questões que se prendem com falta de tempo e também de conhecimentos e prática acerca do tema e da sua abordagem..$^{3,49-10} \mathrm{Em}$ Harsh V. et al..$^{11}$ é referido que o tema pode nunca surgir se a responsabilidade de o introduzir na consulta for dada ao doente..$^{11}$ Diferenças socioeconómicas, culturais, étnicas e etárias podem também dificultar a comunicação sobre a sexualidade. ${ }^{10}$ 
Ao prestarem cuidados de saúde primários, os médicos de família estão numa posição favorável para detectarem problemas relacionados com a vida sexual feminina, implementar estratégias para estabelecer um diagnóstico e iniciar terapêutica quando adequado. ${ }^{9,12} \mathrm{~A}$ consulta de Planeamento Familiar/Saúde da Mulher poderá ser um espaço privilegiado para a abordagem da sexualidade das utentes, atendendo a que se cria um ambiente de maior privacidade e onde também outros aspectos do foro íntimo são focados.

Percebendo as barreiras à comunicação sobre a sexualidade poder-se-ão criar estratégias que permitam ultrapassá-las e assim promover a detecção precoce e tratamento da disfunção sexual na mulher, melhorando a qualidade de vida das utentes. ${ }^{3,7,9}$ Com este trabalho pretendemos identificar algumas dessas barreiras e perceber a relevância do tema para as utentes da consulta de Planeamento Familiar/Saúde da Mulher da Unidade de Saúde Familiar (USF) do Castelo.

\section{MÉTODOS}

Realizou-se um estudo transversal, descritivo, através do auto-preenchimento de questionários pelas mulheres com 18 ou mais anos frequentadoras das consultas de Planeamento Familiar/Saúde da Mulher da USF do Castelo, em Sesimbra. A população de utentes seguidas neste programa de saúde totalizava 1546 doentes. A amostra foi obtida por método não aleatório, consecutivo durante dois meses, de 15 de Março a 15 de Maio de 2010. Tendo em conta o número de marcações por dia nesta consulta, esperávamos obter resposta a cerca de 200 questionários. O questionário foi desenvolvido especificamente para este estudo, contendo 15 questões do tipo pergunta fechada e com várias opções de resposta (Anexo I). Um grupo de questões tinha como objectivo caracterizar as variáveis sócio-demográficas da amostra (idade, nacionalidade, nível de escolaridade e estado conjugal). Outro grupo incidia sobre as variáveis específicas do estudo (grau de satisfação atribuído à vida sexual, percepção da existência de problemas na vida sexual, importância atribuída à abordagem da vida sexual na consulta com o médico de família, frequência com que o médico aborda o tema e com que frequência este é introduzido na consulta pela utente, influência do género do médico na abordagem do tema, razões apontadas pelas uten- tes que dificultam a abordagem do tema na consulta) a partir das restantes questões. O questionário foi testado durante uma semana, tendo sido reformuladas duas perguntas.

Após solicitada a sua participação no estudo, as utentes preencheram o questionário de forma autónoma e confidencial na sala de espera da consulta de Planeamento Familiar/Saúde da Mulher. Foram considerados para o estudo os questionários correcta e completamente preenchidos por utentes com 18 ou mais anos.

A base de dados e tratamento estatístico foram efectuados no programa Microsoft ${ }^{\circledR}$ Office Excel 2007.

\section{RESULTADOS}

Dos 170 questionários preenchidos, nove foram excluídos, tendo sido analisados 161. No que se refere à caracterização desta amostra (Quadro I), podemos ob-

\begin{tabular}{|c|c|c|}
\hline \multicolumn{3}{|c|}{$\begin{array}{l}\text { QUADRO I. Caracterização sócio-demográfica da } \\
\text { amostra estudada }\end{array}$} \\
\hline & n (161) & Percentagem (\%) \\
\hline \multicolumn{3}{|l|}{ Idade (anos) } \\
\hline $18-29$ & 50 & $31,05 \%$ \\
\hline $30-39$ & 49 & $30,43 \%$ \\
\hline $40-49$ & 41 & $25,47 \%$ \\
\hline$\geq 50$ & 21 & $13,05 \%$ \\
\hline \multicolumn{3}{|l|}{ Nacionalidade } \\
\hline Portuguesa & 152 & $94,44 \%$ \\
\hline Outras & 9 & $5,56 \%$ \\
\hline \multicolumn{3}{|l|}{ Nível de escolaridade } \\
\hline Ensino primário & 32 & $19,88 \%$ \\
\hline Ensino básico (9.ªno) & 50 & $31,06 \%$ \\
\hline Ensino secundário (12. ano) & 55 & $34,16 \%$ \\
\hline Ensino Superior & 24 & $14,91 \%$ \\
\hline \multicolumn{3}{|l|}{ Estado conjugal } \\
\hline Solteira & 31 & $19,25 \%$ \\
\hline Casada & 95 & $59,01 \%$ \\
\hline União de facto & 26 & $16,15 \%$ \\
\hline Divorciada & 7 & $4,35 \%$ \\
\hline Viúva & 2 & $1,24 \%$ \\
\hline
\end{tabular}




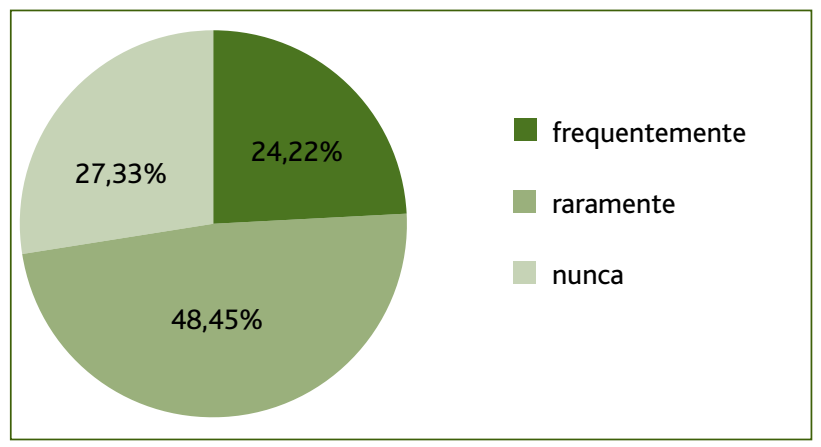

Figura 1. Perspectiva das utentes quanto à frequência da indagação do médico sobre a sua vida sexual.

servar que se distribui de forma homogénea pelos intervalos de idades considerados, sendo a média de idades de 36,6 anos, com uma idade mínima pré-estabelecida de 18 anos e máxima observada de 66 anos. A caracterização sócio-demográfica da amostra encontra-se no Quadro I.

Quando questionadas acerca da sua vida sexual, $50,9 \%$ das inquiridas classifica-a como boa, $35,4 \%$ como satisfatória e 2,5\% como má. Quanto à abordagem da vida sexual na consulta, $75,8 \%$ das mulheres consideram-na importante, sendo que $19,3 \%$ dizem que o fazem frequentemente e $26,7 \%$ referem nunca o ter feito. Por sua vez, $24,2 \%$ afirmam que o médico as ques- tiona frequentemente acerca da sua vida sexual e $48,5 \%$ dizem ser questionadas raramente (Figura 1). Referem nunca ter sido inquiridas acerca do assunto na consulta $27,3 \%$ das mulheres.

Em relação ao facto do género do médico influenciar o seu à-vontade para abordar a sexualidade na consulta, as utentes que participaram no estudo dividem-se, sendo que $50,3 \%$ afirma que não influencia e $49,1 \%$ responde que sim.

No que respeita às razões apontadas como barreiras à abordagem deste tema na consulta destacam-se a vergonha por parte das utentes $(42,5 \%)$, o receio de que $o$ médico não considere o tema importante $(12,6 \%)$ e a falta de tempo na consulta, seleccionada por $10,3 \%$ das inquiridas (Figura 2). De referir que 25,3\% responderam que não existem obstáculos à abordagem da sexualidade na consulta.

\section{DISCUSSÃO}

Neste estudo, os problemas na vida sexual das mulheres surgem numa percentagem inferior à encontrada no estudo de Jan $L$ Shifren, ${ }^{14}$ em que $12 \%$ das mulheres referem desconforto na sua vida sexual. É também bastante inferior à prevalência de disfunção sexual feminina nos EUA descrita por Laumann et al ${ }^{4}(43 \%)$. Salienta-se a percentagem de mulheres que respondeu

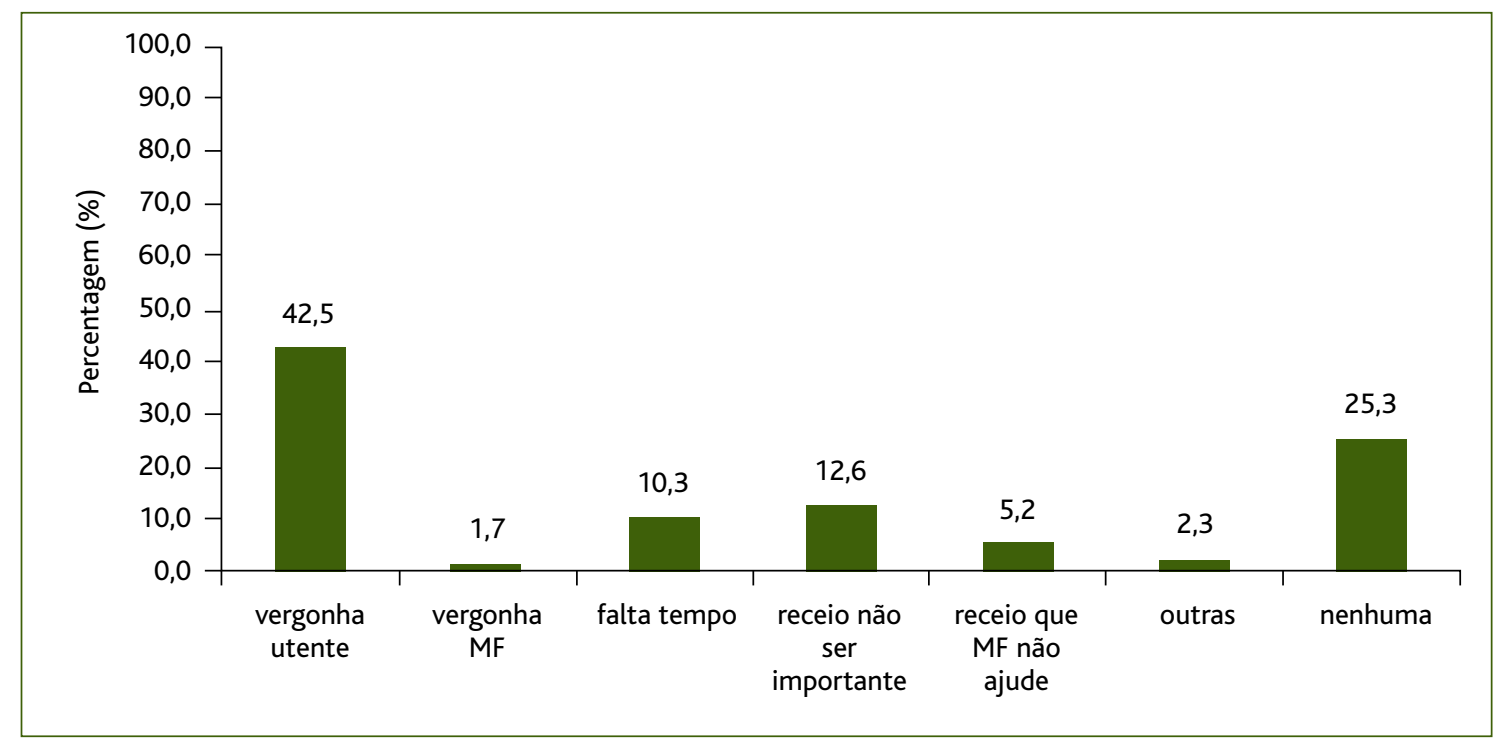

Figura 2. Obstáculos à abordagem da sexualidade na consulta. 
«não sei» quando confrontada com esta questão, $12,4 \%$, um valor elevado e não considerado em outros estudos.

Muitas mulheres, $50,9 \%$, classificam como boa a sua vida sexual, valor não muito diferente dos $44 \%$ encontrados em outro estudo..$^{15}$

Em estudos publicados ${ }^{7-8}$ apenas $14 \%$ das mulheres abordam espontaneamente os problemas da sua vida sexual com o médico, valor próximo do encontrado nas utentes da USF do Castelo analisadas, em que 19,25\% revelam falar frequentemente deste assunto na consulta.

As causas mais frequentemente apontadas pelas utentes como barreiras à abordagem da vida sexual na consulta são a vergonha por parte destas e o receio de que o médico não considere o assunto importante. Estas causas são também frequentemente apontadas em outros estudos. ${ }^{2-3,7,12-13}$ Marwick C. ${ }^{1}$ descreve que $68 \%$ dos doentes receia que a conversa sobre os seus problemas sexuais envergonhe o médico e $71 \%$ acreditam que o médico não dará importância ao seu problema. No nosso estudo as percentagens foram mais baixas mas são também estas as razões mais apontadas pelas utentes. A falta de tempo na consulta é outra razão apontada sendo também um dos motivos referidos em outros estudos sobre sexualidade. ${ }^{6,7}$

Como limitações deste estudo podem-se apontar: utilização de um questionário não validado e o facto de a amostra ser de conveniência e não representativa.

Seria importante averiguar, em eventuais estudos futuros, o ponto de vista dos médicos e enfermeiros que actuam nos Cuidados de Saúde Primários, a fim de apurar outros possíveis entraves à comunicação sobre a sexualidade.

A maioria das mulheres considera importante discutir os problemas da sua vida sexual nas consultas médicas. Ao analisarmos e discutirmos os resultados obtidos neste estudo identificámos as principais barreiras apontadas pelas utentes da USF do Castelo que dificultam a inserção do tema na consulta. Concluímos então que é necessário que o médico mostre à doente que existe à-vontade, disponibilidade e necessidade de abordar este assunto nas consultas.

\section{REFERÊNCIAS BIBLIOGRÁFICAS}

1. Marwick $C$. Survey says patients expect little physician help on sex. JAMA 1999 Jun 16; 281 (23): 2173-4.

2. Hutcherson $\mathrm{H}$. Incorporating sexual medicine into clinical practice. Menopause Management 2006 July-Aug; 15 (4):18-40

3. Kingsberg SA. Identifying HSDD in the family medicine setting. J Fam Pract 2009 Jul; 58 (7 Suppl Hypoactive): 522-5.

4. Laumann EO, PaikA, Rosen RC. Sexual dysfunction in the United States: prevalence and predictors. JAMA 1999 Feb 10; 281 (6): 537-44.

5. Shifren JL, Monz BU, Russo PA, Segreti A, Johannes CB. Sexual problems and distress in United States women: prevalence and correlates. Obstet Gynecol 2008 Nov; 112 (5): 970-8.

6. Nusbaum MR, Hamilton CD. The proactive sexual health history. Am Fam Physician 2002 Nov 1; 66 (9): 1705-12.

7. Kingsberg S. Just ask! Talking to patients about sexual function. Sexuality Reprod Menopause 2004 Dec; 2 (4): 199-203.

8. Gladu RH. Female sexual dysfunction: classification, physiology, diagnosis and treatment. J Sex Reprod Med 2002; 2 (1): 21-7.

9. Simon JA. Opportunities for intervention in HSDD. J Fam Pract 2009 Jul (7 Suppl Hypoactive): S26-S30.

10. Cope DW. The sexual history and approach to the patient with sexual dysfunction. In: Basow DS, editor. UpToDate. Waltham, MA: UpToDate; 2009.

11. Harsh V, McGarvey EL, Clayton AH. Physician attitudes regarding hypoactive sexual desire disorder in a primary care clinic: a pilot study. J Sex Med 2008 Mar; 5 (3): 640-5.

12. Gott M, Galena E, Hinchliff S, Elford H. "Opening a can of worms": GP and practice nurse barriers to talking about sexual health in primary care. Fam Pract 2004 Oct; 21 (5): 528-36.

13. IsHak WW, Mikhail AA, Amiri SR, Berman LA, Vasa M. Sexual dysfunction. Focus 2005; 3: 520-5.

14. Shifren JL. Sexual dysfunction in women: Epidemiology, risk factors and evaluation. In: Basow DS, editor. UpToDate. Waltham, MA: UpToDate; 2009.

15. Laumann EO, Nicolosi A, Glasser DB, Paik A, Gingell C, Moreira E, et al. Sexual problems among women and men aged $40-80 \mathrm{y}$ : prevalence and correlates identified in the Global Study of Sexual Attitudes and Behaviors. Int J Impot Res 2005 Jan-Feb; 17 (1): 39-57.

\section{CONFLITOS DE INTERESSE}

As autoras declaram não terem conflitos de interesse.

\section{ENDEREÇO PARA CORRESPONDÊNCIA}

Ana Lúcia Roça Ramalheiro

USF do Castelo - Estrada do Casalão, Santana, 2970-481 Sesimbra

alramalheiro@gmail.com

Recebido em 16/08/2010

Aceite para publicação em 29/11/2011 


\section{ABSTRACT}

\section{WOMEN'S ATTITUDES TO ATTENTION TO SEXUALITY IN PRIMARY CARE CONSULTATIONS}

Objective: To identify women's attitudes to attention to sexuality in women's health consultations in the Castelo Family Health Unit (USF do Castelo), in order to develop strategies to promote attention to this issue.

Study type: Cross-sectional study.

Setting: USF do Castelo - ACES Seixal e Sesimbra

Population: Women over 18 years of age, attending woman's health consultations in USF do Castelo

Methods: Anonymous questionnaires were distributed to women attending woman's health consultations between March 15th and May 15th, 2010. Data entry and analysis were performed using Microsoft ${ }^{\circledR}$ Office Excel 2007.

Results: Completed questionnaires were received from 161 women. In 5\%, women referred to sexual problems. In the sample, $76 \%$ consider it important to address sexuality in the medical consultation, but only $19.2 \%$ discuss this frequently. Barriers identified include embarrassment (42.3\%), fear that the doctor finds the theme unimportant (12.4\%) and lack of time in the consultation (10.3\%).

Conclusions: Given the barriers identified by patients of USF do Castelo to the discussion of sexuality in the consultation, we suggest that doctors should demonstrate confidence and willingness to discuss sexuality with their patients.

Keywords: Sexuality; Primary Health Care; Female. 


\section{ANEXO I \\ QUESTIONÁRIO}

\section{ABORDAGEM DA VIDA SEXUAL DAS UTENTES NA CONSULTA DE PLANEAMENTO FAMILIAR}

Nota: Este questionário é anónimo e confidencial. Tem como objectivo compreender a comunicação entre médicos e utentes sobre a sexualidade. Por favor, responda a todas as questões com clareza e sinceridade.

1. Idade

2. Nacionalidade

3. Nível de instrução

Ensino primário

Ensino básico ( $9^{\circ}$ ano)

Ensino secundário $\left(12^{\circ}\right.$ ano)

Ensino superior

4. Estado conjugal

Solteira

Casada

União de facto

Divorciada

Viúva

5. Tem vida sexual activa?

Sim

Não

6. Como classifica a sua vida sexual?

Boa

Satisfatória

Má

Não tem vida sexual activa

7. Considera que tem algum problema a nível sexual?
$\square$ Sim
$\square$ Não
Não sei

8. Considera importante falar da sua vida sexual com o/a seu/sua médico/a de família?
Sim
Não
Não tem opinião

9. O/A seu/sua médico/a de família costuma questioná-la acerca da sua vida sexual?

$\square$ Frequentemente

$\square$ Raramente

$\square$ Nunca

10. Costuma colocar questões sobre a sua vida sexual ao/à seu/sua médico/a de família?

$\square$ Frequentemente

$\square$ Raramente

$\square$ Nunca

11. Sente-se à vontade para falar da sua vida sexual com o/a seu/sua médico/a de família?
$\square \operatorname{Sim}$

$\square$ Não

12. O seu médico de família é:

$\square$ Homem

13. Acha que o sexo do/a seu/sua médico/a influencia o seu àvontade para falar da sua vida sexual na consulta?

$\square$ Sim

$\square$ Não

14. Quem é que considera que deve iniciar a conversa sobre a vida sexual na consulta?
$\square$ A utente
O/A médico/a
Indiferente

15. Do seu ponto de vista, quais as razões que dificultam a abordagem deste tema na consulta?

A utente sente vergonha ao falar no assunto

$\square$ A utente receia que o/a médico/a fique envergonhado/a ao falar no assunto

Falta de tempo na consulta

$\square$ Receio que o/a médico/a não considere o assunto importante

$\square$ Receio que o/a médico/a não a possa ajudar

$\square$ Outra

Nenhuma

OBRIGADA PELA SUA COLABORAÇÃO! 